\title{
Phosphoglyceride crystal deposition disease in the abdominal wall: a case report
}

\author{
Osamu Nakahara ${ }^{1 *}$ and Hideo Baba ${ }^{2}$
}

\begin{abstract}
Background: Phosphoglyceride crystal deposition disease (PGDD) is characterized by phosphoglyceride crystal deposition that simulates neoplasia in soft tissue scars or bone. Reports of PGDDs are rare. Here, we present the case of a patient with PGDD in the abdominal wall.

Case presentation: A 57-year-old Japanese man with worsening right lower abdominal pain had no significant family or occupational history. Laboratory data showed elevated inflammatory markers with a white blood cell count of $14,400 \times 10^{9} / \mathrm{L}$ and $\mathrm{C}$-reactive protein of $11.8 \mathrm{mg} / \mathrm{L}$, but no other abnormalities. Helical computed tomography (CT) revealed a tumor in the abdominal wall (longest dimension, approximately $10 \mathrm{~cm}$ ). Positron emission tomography-CT revealed fluorodeoxyglucose accumulation in the mass only (SUVmax, 41). Clinical and radiographic findings suggested malignant lymphoma, undifferentiated sarcoma, or liposarcoma. He underwent exploratory laparotomy and further treatment. At surgery, we found a huge milky-whitish mass with a rough surface in the transversus abdominis. Complete resection was performed and his postoperative recovery was good. Surprisingly, the final pathologic diagnosis was phosphoglyceride crystal deposition disease with the characteristic crystal deposition in a corolla shape, histiocytic reaction with abundant foreign-body-type giant cells, and no evidence of neoplasia. The patient remains asymptomatic with no disease recurrence.

Conclusion: Although phosphoglyceride crystal deposition disease in the abdominal wall is rarely encountered in clinical practice, its inclusion in differential diagnosis is important. Given the occurrence at sites of invasive procedures, we believe efforts to reduce invasiveness when performing surgery and follow-up for early detection of recurrence are important.
\end{abstract}

Keywords: Soft tissue tumor, Phosphoglyceride crystal deposition disease, Foreign-body granuloma

\section{Background}

Phosphoglyceride crystal deposition disease (PGDD) is characterized by phosphoglyceride crystal deposition that simulates neoplasia in soft tissue scars or bone. The condition is rarely encountered in the clinical setting and is sometimes strongly suspected of being a malignant tumor based on clinical and radiographic findings. The lesions often occur over injection sites or surgical scars, such as the periumbilical area or upper arm, suggesting a possible relationship with these procedures.

\footnotetext{
* Correspondence: o-naka@live.jp

${ }^{1}$ Department of Surgery, Taragi Municipal Hospital, 4210 Taragi, Taragi-machi, Kuma-gun, Kumamoto 868-0598, Japan

Full list of author information is available at the end of the article
}

In this report, we describe a case of PGDD in the abdominal wall, which was resected surgically after being initially diagnosed as a malignant tumor.

\section{Case presentation}

A 57-year-old Japanese man was referred to our hospital because of increasing right lower abdominal pain. He had a history of appendectomy at the age of 17 years with no significant family or occupational history.

On initial examination, an abdominal wall tumor (largest dimension, approximately $10 \mathrm{~cm}$ in diameter) was detected using transabdominal ultrasound (Fig. 1). Laboratory data revealed elevated inflammatory markers $\left(\mathrm{WBC}=14,400 \times 10^{9} / \mathrm{L}, \mathrm{CRP}=11.8 \mathrm{mg} / \mathrm{L}\right)$; major tumor markers (carcinoembryonic antigen, CA19-9, and soluble 


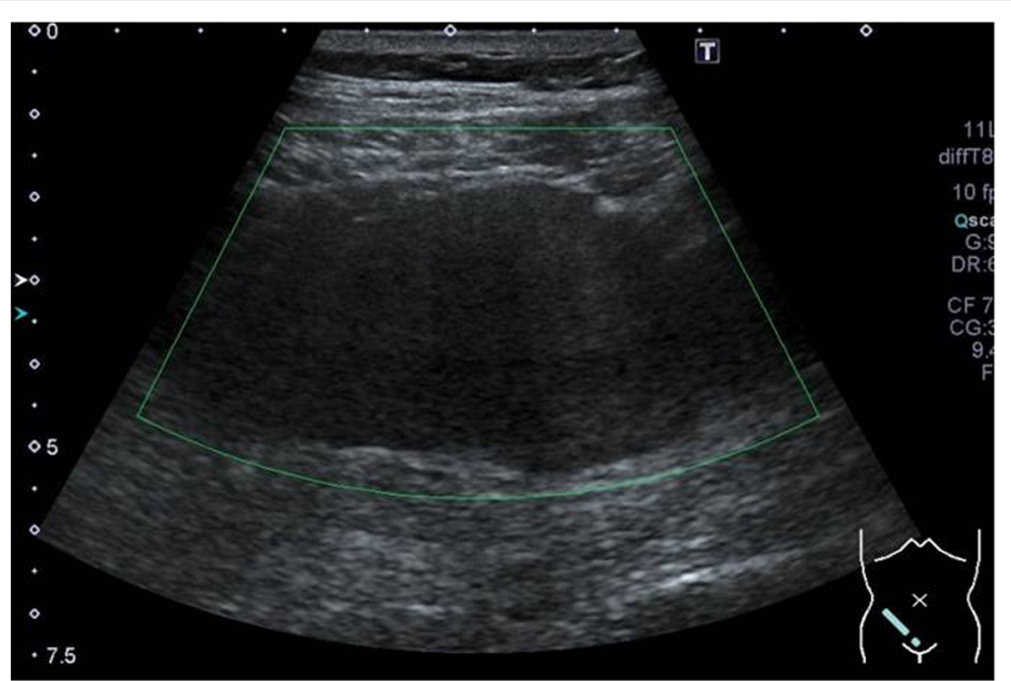

Fig. 1 Transabdominal ultrasonographic image of a tumor measuring $10 \mathrm{~cm}$ in diameter showing a homogeneous echo pattern

IL-2 receptor) were within normal limits. Helical computed tomography (CT) also revealed a solid mass (largest dimension, $10 \mathrm{~cm}$ in diameter) in the abdominal wall (Fig. 2). Magnetic resonance imaging showed a mass that exhibited low intensity on T2-weighted images, slightly high intensity on diffusion-weighted images, and gradual reinforcement on dynamic study (Fig. 3). Positron emission tomography-CT revealed fluorodeoxyglucose accumulation in the mass only (SUVmax, 41) (Fig. 4). Because clinical and radiographic findings suggested malignant lymphoma, undifferentiated sarcoma, or liposarcoma, he underwent exploratory laparotomy and treatment.



Fig. 2 Helical computed tomography (CT) indicating a solid mass in the abdominal wall (arrow)
Intraoperative findings at laparotomy were an elastic, hard, milky-whitish mass with a rough surface and capillary growth in the right lower quadrant (Fig. 5). On inspection and palpation, a malignant tumor was strongly suspected. No other tumor suspicious of a primary lesion was found in the intraabdominal organs, including the gastrointestinal tract. The mass was completely removed, and the surgical margin secured. The total weight of the mass was $120 \mathrm{~g}$. No complications were observed during the perioperative period, and the patient was discharged on postoperative day 7 .

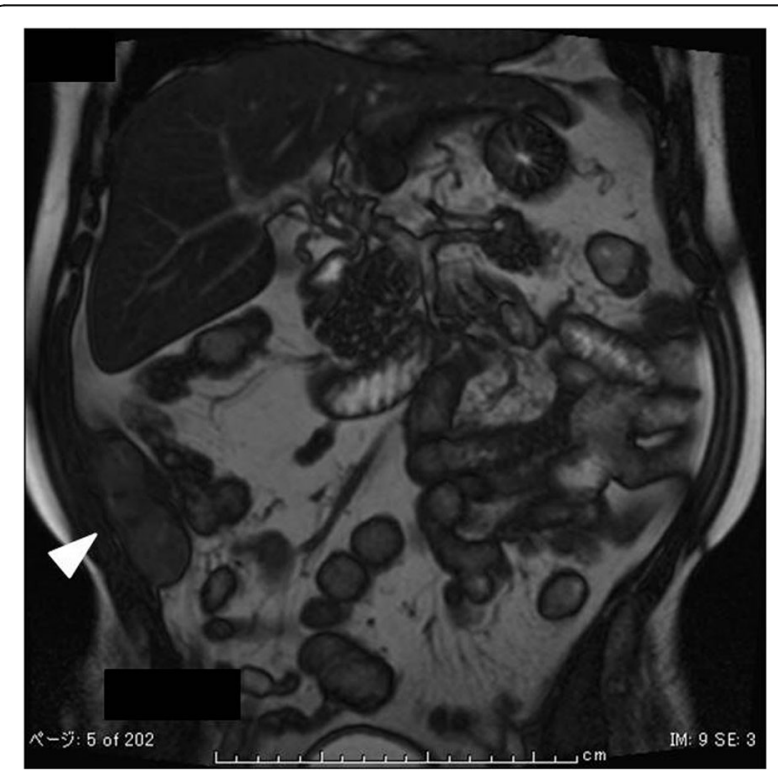

Fig. 3 Magnetic resonance imaging of the abdomen showing elliptical masses in the transverse abdominal wall exhibiting low intensity on T2-weighted images (arrowhead) 




Fig. 4 FDG-PET showing abnormal accumulation (SUVmax 41) in the abdominal wall

Pathologic examination revealed that these masses were foreign-body granulomas consisting of string-like crystals and a foreign-body giant cell (Fig. 6a, b). Immunohistological staining using anti-CD68 antibody (clone KP1) against the CD68 antigen, which is a known macrophage



Fig. 5 Intraoperative abdominal view of an elastic, hard, milky-white tumor with a rough surface. Capillary development is evident in the right lower quadrant surface marker, was positive in cells surrounding phosphoglyceride crystals (Fig. 6c).

The patient underwent regular follow-up abdominal ultrasound examination and CT postoperatively. As of 3 years after the procedure, no signs of recurrence have been detected.

PGDD is a rare disease characterized by deposition of phosphoglyceride crystals, often simulating neoplasm in a scar of soft tissue or bone. It can sometimes form a large mass and be misdiagnosed as a malignant tumor.

Little is known about its etiology and pathogenesis. This deposition disease is apparently triggered by injury and subsequent macrophage aggregation, with a histological picture of deposited crystal radiating from the cell membranes of epithelioid cells. The macrophages themselves may contribute to the formation of a nidus for the crystals. One hypothesis is that localized disturbance of phosphoglyceride metabolism within the macrophages may be initiated by local inflammation, leading to progressive amplification of macrophage infiltration and crystal deposition.

To our knowledge, only 10 cases of PGDD have been reported previously, including our case. The available clinical information on these cases is summarized in Table 1 [1-7]. The mean age of patients is 57 (range, 37-76) years. There appears to be no gender predilection. No congenital abnormalities or family history of metabolic disorders have been found. Deposition was characteristically noted at intramuscular injection sites or postoperative sites like the gluteal muscles and the deltoid, abdominal wall soft tissue, scapula, spine, myocardium, and pelvic soft tissue. The size of the tumors reported is highly variable, from $3.5 \mathrm{~cm}$ to the size of an infant's head, but in general it is a relatively large tumor with a mean size of $8.9 \mathrm{~cm}$. The time from the initial invasion to confirmation of the tumor was at least 20 years and was 45 years in the longest case. About half of the cases had multiple lesions. In most cases, excisional surgery was performed due to local tumor formation without apparent symptoms of inflammation. Tumors that occurred at postoperative sites were suspected to be true malignant neoplasms or recurrent tumors. In one case, intraoperative rapid pathological diagnosis revealed PGDD, so complete resection was not performed and the patient was observed clinically.

No specific markers exist for PGDD, and differentiating this disease from malignant tumors without pathological examination is difficult. We considered needle biopsy in the present case but decided against it given the risk of needle tract seeding if malignant.

In the present case, exploratory laparotomy, not laparoscopic surgery, was performed for a suspected huge malignant tumor of the abdominal wall, based on radiographic examination. Our patient had a history of 

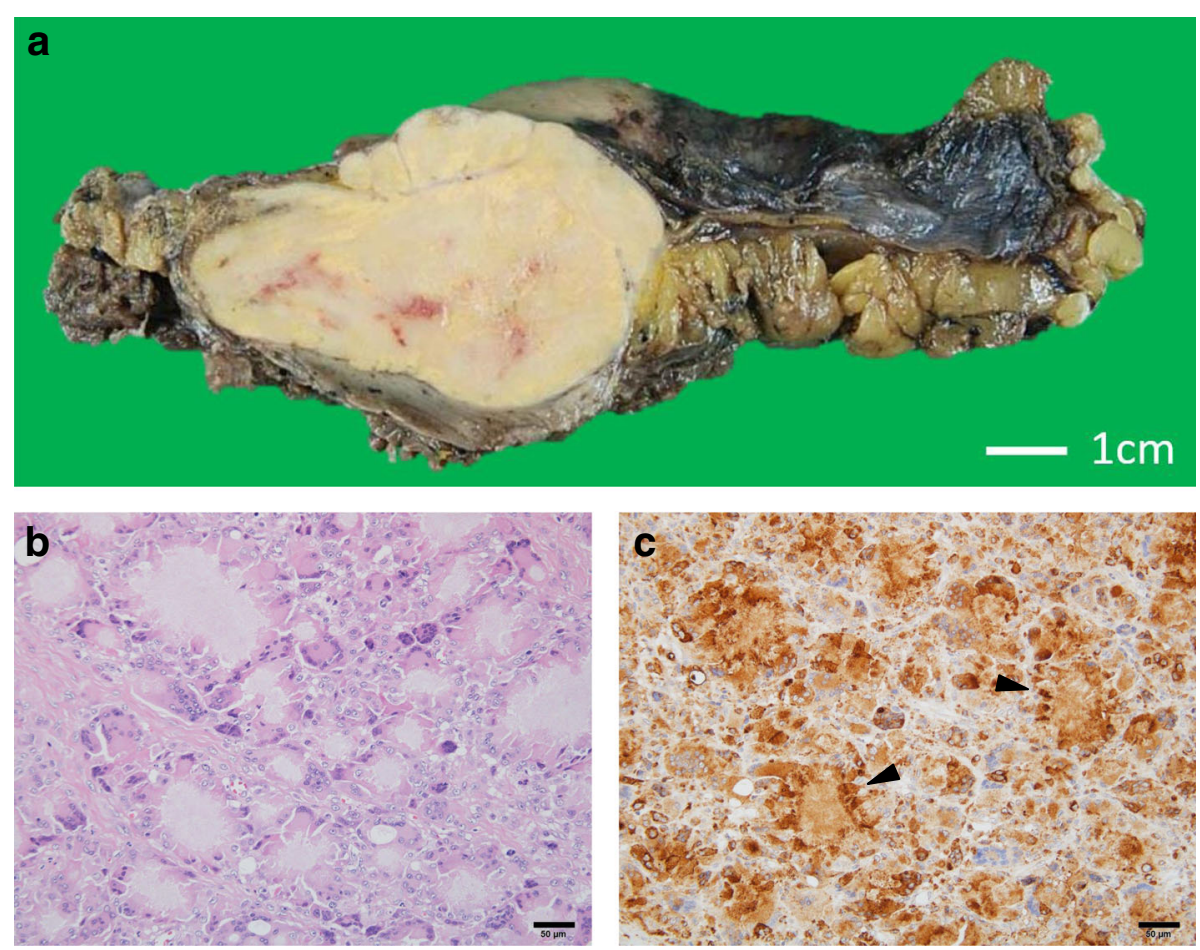

Fig. 6 a Macroscopic findings of the cut surface of the tumoral mass showing a lobulated, firm, yellowish-white portion. b Histological findings. Hematoxylin and eosin staining showing a foreign-body granuloma consisting of string-like crystals and a foreign-body giant cell. Many tiny granulomas with central bluish-pink crystals surrounded by macrophages are evident. Bar $50 \mu \mathrm{m}$. c Immunohistological staining using anti-CD68 antibody (clone KP1) against CD68, a known macrophage surface marker, was positive in cells surrounding phosphoglyceride crystals (arrowhead) bar $50 \mu \mathrm{m}$

appendectomy at the age of 17 years, and local inflammation at the operation site was suspected to have led to progressive amplification of macrophage infiltration and crystal deposition.

Postoperative follow-up has not revealed any findings suspicious of recurrence in our case. However, there are reports in the literature of recurrence at 4 years after surgery [7]. Few studies have reported on the process of crystallization and deposition of phosphoglycerides and so the mechanism remains unclear. Local injection of a bovine-derived medication has been suggested as causative, yet that alone does not explain PGDD, including the present case. In reviewing previous reports, a few cases suggest that during the process of phosphoglyceride metabolism, a locally invasive procedure increases macrophage activation and leads to phosphoglyceride

Table 1 Clinical summary of phosphoglyceride crystal deposition disease

\begin{tabular}{|c|c|c|c|c|c|c|c|}
\hline Age & Gender & Location & Past medical history & Interval period & No. of tumors & Tumor size & Reference \\
\hline 58 & M & Buttock muscle & Not detected & Not detected & Single & $11 \mathrm{~cm}$ & Kubo, $1992[1]$ \\
\hline \multirow[t]{3}{*}{62} & $\mathrm{~F}$ & Oral soft tissue & Appendectomy, dental treatment & 20 years over & Multiple & $11 \mathrm{~cm}$ & Miura, 2000 [2] \\
\hline & & Abdominal soft region & & & & & \\
\hline & & Brachial muscle & & & & & \\
\hline 51 & M & Abdominal soft region & Post-gastrectomy & 33 year & Multiple & $3.5 \mathrm{~cm}$ & Yachida, 2002 [3] \\
\hline 58 & M & Abdominal soft region & Post-gastrectomy & 40 years over & Single & Infant head size & Miura, 2004 [4] \\
\hline 64 & $\mathrm{~F}$ & Abdominal soft region & Post-gastrectomy & 35 year & Single & $4 \mathrm{~cm}$ & Miura, 2004 \\
\hline 64 & $\mathrm{~F}$ & Scapular bone & Not detected & Not detected & Single & $10 \mathrm{~cm}$ & Miura, 2004 \\
\hline 76 & $\mathrm{~F}$ & Spine & Lumbar anesthesia & 45 year & Multiple & Not detected & Nishimura, 2005 [5] \\
\hline 37 & M & Anterior mediastinum & Ventricular septal defect & 35 year & Single & $6 \mathrm{~cm}$ & Shoji, 2007 [6] \\
\hline 50 & $\mathrm{~F}$ & Pelvic soft tissues & Cesarean delivery & 26 year & Multiple & $10 \mathrm{~cm}$ & Yamada, 2015 [7] \\
\hline 57 & M & Abdominal soft region & Appendectomy & 40 year & Single & $10 \mathrm{~cm}$ & Our case \\
\hline
\end{tabular}


deposition. Moreover, knowledge of the tendency of PGDD to form at sites of invasion may allow a clinician to clinically observe a similar mass found during routine follow-up, and debulking surgery may be considered as a therapeutic option for symptomatic cases.

\section{Conclusions}

PGDD is extremely rare but should be considered in the differential diagnosis of abdominal tumors in patients with a history of abdominal surgery. Because PGDD results from inflammatory response at sites of locally invasive procedures, multiple lesions and recurrence are possible and careful observation of the clinical course is therefore important.

\section{Abbreviations}

CT: Computed tomography; PGDD: Phosphoglyceride crystal deposition disease

\section{Funding}

This research received no specific grant from any funding agency in the public, commercial, or not-for-profit sectors.

\section{Authors' contributions}

$\mathrm{ON}$ and $\mathrm{HB}$ collected, analyzed, and interpreted the data and revised the manuscript. Both authors read and approved the final manuscript.

\section{Ethics approval and consent to participate}

This study was carried out in accordance with the principles of the Declaration of Helsinki.

\section{Consent for publication}

Written informed consent was obtained from the patient for publication of this case report and any accompanying images.

\section{Competing interests}

The authors declare that they have no competing interest.

\section{Publisher's Note}

Springer Nature remains neutral with regard to jurisdictional claims in published maps and institutional affiliations.

\section{Author details}

'Department of Surgery, Taragi Municipal Hospital, 4210 Taragi, Taragi-machi, Kuma-gun, Kumamoto 868-0598, Japan. ${ }^{2}$ Department of Gastroenterological Surgery, Graduate School of Medical Sciences, Kumamoto University, 1-1-1

Honjo, Chuo-ku, Kumamoto City, Kumamoto 860-8556, Japan.

Received: 5 March 2018 Accepted: 27 August 2018

Published online: 19 September 2018

\section{References}

1. Kiyoko Kubo JM, Matsumoto S, Kawaguchi T, Kitagawa T. Unusual huge intramuscular granuloma with calcium phosphate crystal deposition in the buttock. Acta Pathol Jpn. 1992:42:508-11.

2. Miura K, Fukuda H, Mineta H, Yamaguchi K, Harada H, Yusa H, Tsutsui Y. Phosphoglyceride crystal deposition disease. Pathol Int. 2000;50(12):992-8.

3. Yachida SFN, Nakanishi Y, Nimura S, Sato M, Shimada K, Miura K, Sakamoto M. Phosphoglyceride crystal deposition disease presenting as multiple paragastric masses. Dig Dis Sci. 2002;47:1413-8.

4. Miura K, Han G, Kondo S, Ohhashi H, Tsutsui Y. Phosphoglyceride (PG) crystal deposition disease: a novel acquired disease in which PG crystals are deposited in injured soft tissue and bone with phospholipid metabolism disturbance. Pathol Int. 2004;54(12):881-9.

5. Nishimura A, Kasai Y, Takegami K, Uchida A. Phosphoglyceride crystal deposition disease in the spine: a case report. J Spinal Disord Tech. 2005; 18(1):112-5.
6. Shoji F, Yoshino I, Kometani T, Yamamoto H, Maehara Y. Phosphoglyceride crystal deposition disease originating from the myocardium. J Thorac Cardiovasc Surg. 2007;134(2):508-9.

7. Yamada Y, Nishioka K, Kajihara H, Noguchi T, Naruse K, Horie K. A case of phosphoglyceride crystal deposition disease in the pelvic soft tissues recurring after initial surgery. Case Rep Obstet Gynecol. 2015;2015:751582.

\section{Submit your manuscript to a SpringerOpen ${ }^{\circ}$ journal and benefit from:}

- Convenient online submission

- Rigorous peer review

- Open access: articles freely available online

High visibility within the field

- Retaining the copyright to your article

Submit your next manuscript at $\boldsymbol{\nabla}$ springeropen.com 